\title{
Estimate of tilt instability of mesa-beam and Gaussian-beam modes for advanced LIGO
}

\author{
Pavlin Savov \\ Theoretical Astrophysics; California Institute of Technology, Pasadena, California, USA
}

Sergey Vyatchanin

MSURG LSC Group; Physics Department, Moscow State University, Moscow, Russia

(Received 21 September 2004; revised manuscript received 15 August 2006; published 9 October 2006)

\begin{abstract}
Sidles and Sigg have shown that advanced LIGO interferometers will encounter a serious tilt instability, in which symmetric tilts of the mirrors of an arm cavity cause the cavity's light beam to slide sideways, so its radiation pressure exerts a torque that increases the tilt. Sidles and Sigg showed that the strength $T$ of this torque is 26.2 times greater for advanced LIGO's baseline cavities - nearly flat spherical mirrors which support Gaussian beams (FG cavities), than for nearly concentric spherical mirrors which support Gaussian beams (CG cavities) with the same diffraction losses as the baseline case: $T^{\mathrm{FG}} / T^{\mathrm{CG}}=26.2$. This has motivated a proposal to change the baseline design to nearly concentric, spherical mirrors. In order to reduce thermal noises in advanced LIGO, O'Shaughnessy and Thorne have proposed replacing the spherical mirrors and their Gaussian beams by "Mexican-Hat" (MH) shaped mirrors which support flattopped, mesa shaped beams. In this paper, we compute the tilt-instability torque for advanced-LIGO cavities with nearly flat MH mirrors and mesa beams (FM cavities) and nearly concentric MH mirrors and mesa beams (CM cavities), with the same diffraction losses as in the baseline FG case. We find that the relative sizes of the restoring torques are $T^{\mathrm{CM}} / T^{\mathrm{CG}}=0.91, T^{\mathrm{FM}} / T^{\mathrm{CG}}=96, T^{\mathrm{FM}} / T^{\mathrm{FG}}=3.67$. Thus, the nearly concentric $\mathrm{MH}$ mirrors have a weaker tilt instability than any other configuration. Their thermoelastic noise is the same as for nearly flat $\mathrm{MH}$ mirrors, and is much lower than for spherical mirrors.
\end{abstract}

DOI: 10.1103/PhysRevD.74.082002

PACS numbers: 04.80.Nn, 07.60.Ly, 95.55.Ym

\section{INTRODUCTION}

Thermal noises in mirror substrates and mirror coatings are the dominant noise sources for advanced LIGO at and somewhat below the frequency of optimal sensitivity. When sapphire mirrors were planned for advanced LIGO, the dominant thermal noise was thermoelastic substrate noise, and O'Shaughessy and Thorne $[1,2]$ proposed lowering that noise by flattening the cross-sectional profile of the arm cavities' light beams - i.e., by replacing the standard Gaussian-shaped beams by "mesa"-shaped beams (thick curves in Fig. 2 below). This can be achieved by replacing LIGO's nearly flat, spherically shaped mirrors by mirrors that have a nearly flat "Mexican-hat" (MH) shape. More recently, fused silica has been selected as baseline mirror material, and thermoelastic substrate noise is no longer dominant. However, the other three forms of thermal noise (Brownian substrate, Brownian coating, and thermoelastic coating) are also substantially reduced by switching from Gaussian beams and spherical mirrors to mesa beams and Mexican-hat mirrors, so mesa beams remain an attractive possibility for advanced LIGO and/ or for other future interferometers. For detailed computations of the noise reductions achieved by using mesa beams, see O'Shaughessy, Strigin and Vyatchanin [1-3], Agresti [4-6] and Lovelace [7].

Sidles and Sigg [8,9] have recently rediscovered a tilt instability in Fabry-Perot (FP) cavities, first pointed out by Braginsky and Manukin [10], and they have shown that this instability is a serious issue for advanced LIGO's arm cavities, because of their high circulating light power (about $800 \mathrm{~kW}$ ) and resulting high light pressure. In this instability, random forces cause the cavity's mirrors to tilt in a symmetric way ${ }^{1}[$ Fig. 1(b)], and this tilt causes the light beam to slide sideways in the cavity by the distance $\delta x_{\text {sym }}$ shown in the figure, so its light pressure exerts a torque $T$ on the mirrors that tries to increase their tilt. [Sidles and Sigg also showed that, when the mirrors are tilted in an antisymmetric way as in Fig. 1(c), the resulting torque is stabilizing rather than destabilizing.] Sidles and Sigg analyzed the tilt instability, using geometric arguments, for cavities with nearly flat, spherical mirrors and their Gaussian light beams ("FG" cavities), and also for nearly concentric, spherical mirrors and their Gaussian beams ("CG" cavities). [The mirrors must be nearly flat or nearly concentric in order to make the light beams significantly larger than the Fresnel diffraction size, $b=$ $\sqrt{\lambda L / 2} \pi$ with $\lambda$ the light's wavelength and $L$ the cavity length; large beams are required to keep the thermoelastic noise small.] Sidles and Sigg found that the instability is much more severe for the baseline FG cavities than for CG cavities with the same beam radii at the mirrors and then the same diffraction losses. On this basis, the baseline design for advanced LIGO [11] has been changed from FG cavities with nearly flat mirrors to CG cavities with nearly concentric mirrors.

Motivated by this Sidles-Sigg work, Thorne has proposed a mathematical way to design nearly concentric

\footnotetext{
${ }^{1}$ Sidles and Sigg $[8,9]$ use the opposite convention from us for "symmetric and "antisymmetric" tilt.
} 
PAVLIN SAVOV AND SERGEY VYATCHANIN
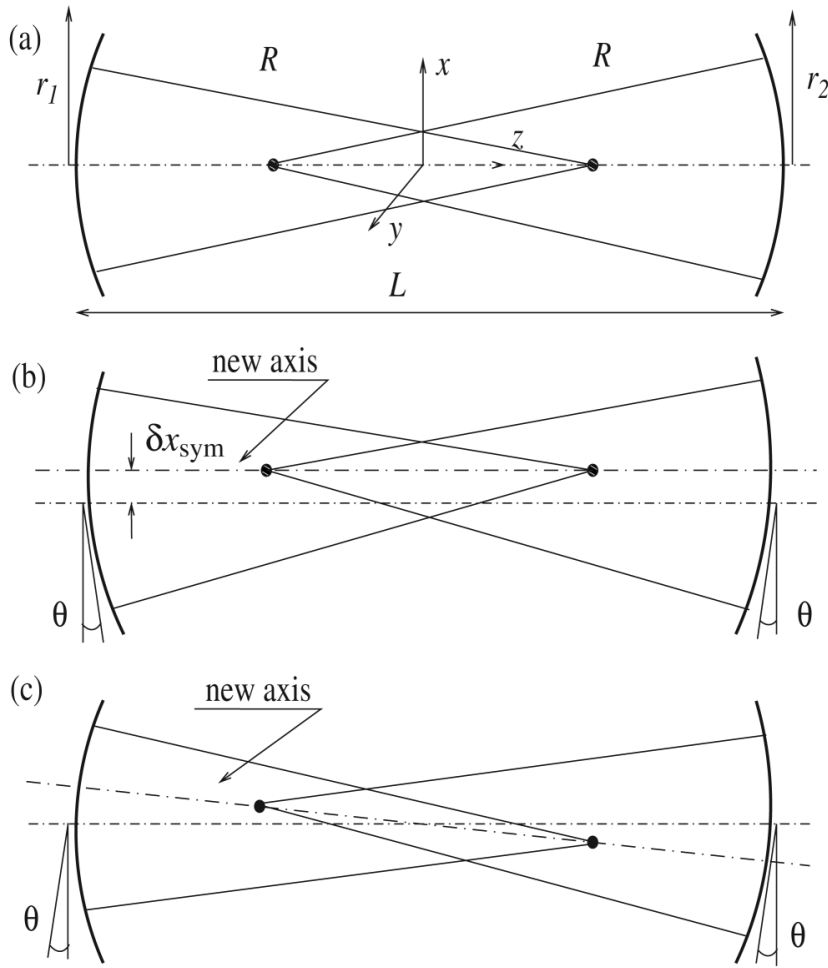

FIG. 1. Horizontal section of FP resonator with (a) perfectly positioned spherical mirrors, (b) symmetrically tilted spherical mirrors and (c) antisymmetrically tilted spherical mirrors.

MH mirrors that support mesa beams with precisely the same mesa-shaped light-power distributions on the mirrors as for the original nearly flat MH mirrors. Thorne's mathematical construction is presented, along with some generalizations of it, in a companion paper by Bondarescu [12].

In the present paper, we analyze the tilt instability for advanced-LIGO arm cavities with (i) nearly flat MH mirrors and their mesa beams ("FM" cavities), and (ii) with Thorne's new nearly concentric MH mirrors and their mesa beams ("CM" cavities). We employ first-order perturbation theory in our analysis, by contrast with the Sidles-Sigg geometric techniques. We compare the strength of the tilt's destabilizing torque $T$ for FG, FM, CG, and CM cavities that have beam sizes chosen so they all have the same diffraction losses, about $20 \mathrm{ppm}$; and we explore two choices for the radius of the mirror coating on the substrates: the baseline radius $(14.7 \mathrm{~cm})$, and a larger coated radius $(16 \mathrm{~cm})$ used in the analysis of d'Ambrosio et al. [1,2] [their fiducial configuration].

In our numerical solutions to the eigenequation for the light's eigenmodes inside FM and CM cavities, we discovered remarkable duality relations between cavities with axisymmetric mirrors that deviate by an amount $H(r)$ from flatness, and cavities with mirrors that deviate by $-H(r)$ from concentric spheres. We verified these numerically discovered duality relations for several different forms of $H(r)$, in addition to those of $\mathrm{MH}$ mirrors. This motivated Chen and Savov, and independently Agresti and
PHYSICAL REVIEW D 74, 082002 (2006)
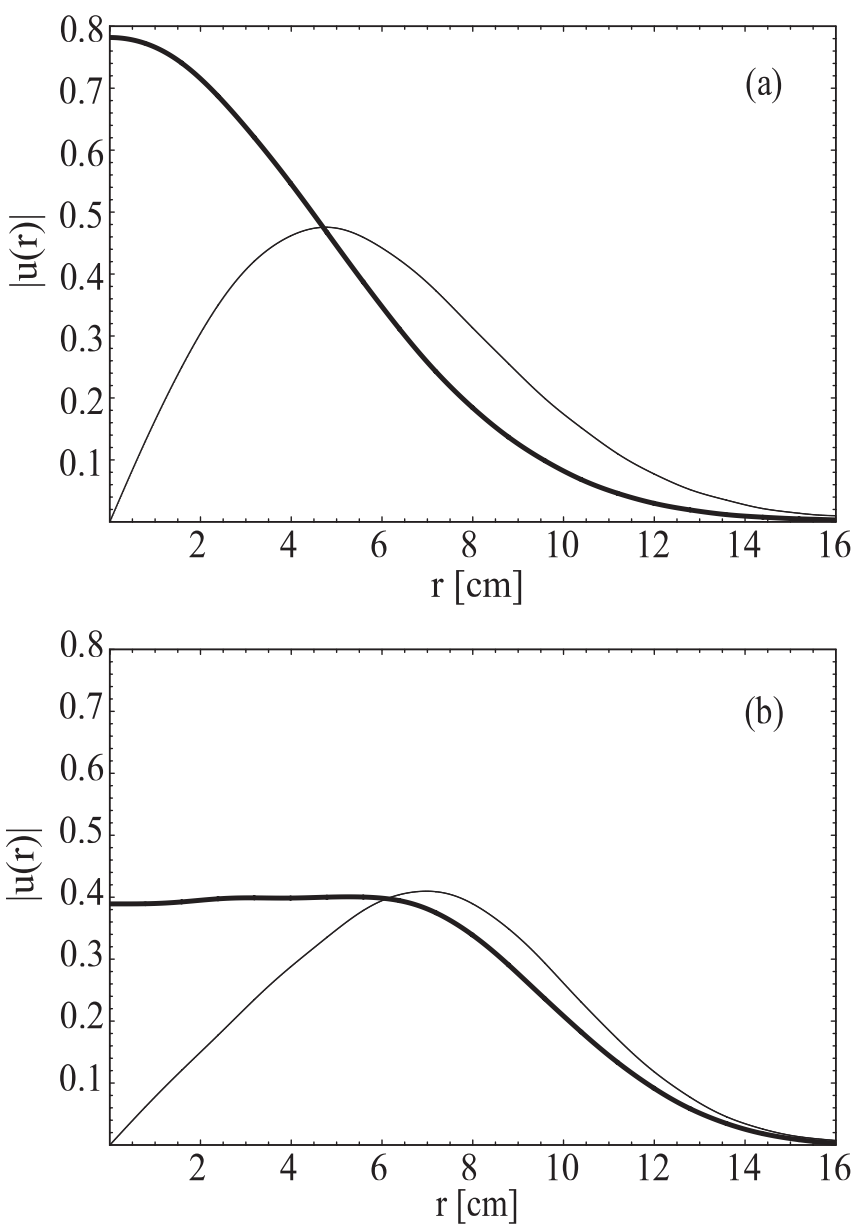

FIG. 2. Fundamental modes $u_{0}(r)$ (thick curves) and first dipolar modes $u_{1}(r)$ (thin curves) at mirrors' surfaces for (a) FG and $\mathrm{CG}$ cavities, and (b) FM and CM cavities. The modes are dimensionless and normalized according to Eqs. (2.11) and (2.12). We have used the fiducial cavity parameters of d'Ambrosio et al.: Eqs. (2) of Sec. IVA of [1] and Sec. IIIA of [2].

d'Ambrosio [13] to devise analytic proofs of our duality relations. The duality relations provide a unique one-toone mapping between the eigenstates and eigenvalues of the dual cavities - a mapping that may be useful not only for advanced LIGO but in a variety of other applications of Fabry-Perot cavities.

This paper is organized as follows. In Sec. II, we use a first-order modal analysis of a Fabry-Perot cavity to derive a general formula for the torque exerted on the mirrors when the cavity is perturbed, in terms of as-yet unknown mode coupling coefficients $\alpha_{k}$ and mode-overlap integrals $I_{k}$. In Sec. III, we use first-order perturbation theory of Gaussian-beam (FG and CG) cavities to derive analytical formulas for $\alpha_{k}$ and $I_{k}$, and then for the tilt-induced torque $T$ in the FG and CG cases, and we show that our formula for the torque is equivalent to that of Sidles and Sigg $[8,9]$. In Sec. IV we use first-order perturbation theory to derive formulas for the coupling coefficients $\alpha_{k}$, and then for the 
torque $T$, in terms of a cavity's eigenvalues and modeoverlap integrals $I_{k}$. In Sec. V we present our numerical results for the modes and their eigenvalues for FM, CM, FG, and CG cavities, and we discuss the duality relations between the nearly flat and nearly concentric cases. Finally, in Sec. VI, we combine the numerical results of Sec. V with the formulas of Secs. III and IV, to deduce the tilt-induced torque for our four cavity designs - using two sets of parameters: those for cavities with advanced-LIGO baseline mirror radii, and those for d'Ambrosio et al.'s slightly larger mirrors ("fiducial" configurations). We present a brief conclusion in Sec. VII. For the readers interested in our numerical implementation of the eigenvalue problem, we include an Appendix where we sketch details of our computational work.

The results presented in this paper are based on previous work on nearly flat configurations by S. Vyatchanin [14] (some errors in this paper are corrected here) combined with recent analyses of nearly concentric cavities by $P$. Savov. An analytical proof of the duality relation between nearly flat and nearly confocal resonators by P. Savov and Y. Chen, and independently by E. D'Ambrosio and J. Agresti, will be provided in a companion paper [13].

\section{MAIN FORMULAS}

The light inside LIGO arm cavities is well-described by the laws of diffraction optics in the paraxial approximation. The eigenvalue problem in this approximation for a half trip through a cavity with two identical axisymmetric mirrors can be written as

$$
\int G\left(\vec{r}_{1}, \vec{r}_{2}\right) u\left(\vec{r}_{2}\right) d^{2} \vec{r}_{2}=\lambda u\left(\vec{r}_{1}\right) .
$$

In the above equation $u(\vec{r})$ is an eigenmode of the cavity and $\lambda$ is the corresponding eigenvalue. The eigenmode represents the state of the light (the electric field) on the surface of a mirror.

For advanced LIGO diffraction losses will be very small (about $10 \mathrm{ppm}$ for each half trip), so it is an excellent idealization to ignore the losses and idealize the mirrors as infinite in radius. Then, $|\lambda|=1, G$ is a unitary operator, and its eigenvectors form a complete set. Each eigenmode $u_{n m}$ and the corresponding eigenvalue $\lambda_{n m}$ are labeled by two (quantum) numbers - radial (or principle) number $n=0,1, \ldots$ and angular (or azimuthal) number $m=$ $0,1, \ldots$ All modes with angular number $m=0$ are axisymmetric (no angular dependence), $m=1$ are dipolar, $m=2$ are quadrupole, etc.:

$$
u_{n m} \propto e^{-\mathrm{im} \varphi} .
$$

The eigenmodes are normalized and orthogonal to each other according to the following definition:

$$
\int u_{n_{1} m_{1}}(\vec{r}) u_{n_{2} m_{2}}^{*}(\vec{r}) d^{2} \vec{r}=\delta_{n_{1} n_{2}} \delta_{m_{1} m_{2}} .
$$

We will use this set of eigenvectors as a basis for expanding the eigenmodes of cavities with tilted mirrors. The radial coordinate $r$ is dimensionless and measured in units of the Fresnel diffraction size

$$
b=\sqrt{L \lambda / 2 \pi} .
$$

When the mirrors of a FP cavity are tilted in a symmetric way [as in Fig. 1(b)], the cavity's fundamental mode $u_{00}(\vec{r})$ is transformed into the fundamental mode $\tilde{u}_{00}(\vec{r})$ of the perturbed cavity. The torque acting on the mirrors when the light is in this mode and has power $P$ is

$$
T=\frac{2 P b}{c} \int\left|\tilde{u}_{00}(\vec{r})\right|^{2} r \cos \varphi d^{2} \vec{r} .
$$

The new fundamental mode can be expanded over the set of orthonormal modes $\left\{u_{n m}(\vec{r})\right\}$ of the unperturbed cavity

$$
\tilde{u}_{00}(\vec{r})=u_{00}(\vec{r})+\sum_{n, m} \alpha_{n m} u_{n m}(\vec{r}) .
$$

In this paper, we study effects only to first order in the perturbation. That is why the coefficient in front of $u_{00}(\vec{r})$, in Eq. (2.6), is unity.

By substituting Eq. (2.6) into Eq. (2.5) and using the angular dependence of the eigenmodes Eq. (2.2), we conclude that only the dipolar eigenmodes $(m=1)$ contribute to the net torque and more specifically their part proportional to $\cos (\varphi)$. Thus, for our purposes of calculating the torque, we will assume $u_{n 1} \propto \cos (\varphi)$. Since the only modes we use from now on are the fundamental mode $u_{00}$ and all dipolar modes $u_{n 1}$, in order to simplify notation, we collapse the indices into one labeling index

$$
k=n+m .
$$

Thus the fundamental mode becomes $u_{0}$, the first dipolar mode becomes $u_{1}$ (corresponding to the old notation $u_{01}$ ) and so on. When necessary, we will use the conventional notation with two labeling indices.

We will study the effects of tilt only to first order in the tilt angle $\theta$, so for our purposes we use the following expansion of the perturbed eigenmode:

$$
\begin{gathered}
\tilde{u}_{0}(\vec{r})=u_{0}(\vec{r})+\sum_{k=1} \alpha_{k} u_{k}(\vec{r}), \\
u_{0}(\vec{r})=\frac{u_{0}(r)}{\sqrt{2 \pi}}, \\
u_{k}(\vec{r})=\frac{u_{k}(r) \cos \varphi}{\sqrt{\pi}}, \\
\int_{0}^{\infty}\left[u_{0}(r)\right]^{2} r d r=1,
\end{gathered}
$$




$$
\int_{0}^{\infty}\left[u_{k}(r)\right]^{2} r d r=1, \quad k=1,2, \ldots
$$

In the above equations, $u_{k}(\vec{r})$ are the dipolar modes on the surface of a mirror; $u_{k}(r)$ are their parts depending only on the radial coordinate $r$; all $u_{k}(r)$ are dimensionless and normalized as shown above [cf. Equation (2.3) with $m=$ $0,1, k=n+m]$; and $\alpha_{k}$ are dimensionless coupling constants, proportional to the mirrors' tilt angle $\theta$, which we will evaluate in Sec. III for Gaussian (FG and CG) beams and in Sec. IV for mesa (FM and CM) beams. In general, $u_{k}(\vec{r})$ are complex fields, but since the mirror surfaces coincide with the beam's wave front, up to an overall complex phase which we chose to be zero, they are real fields.

Now we can calculate the torque that the cavity's light exerts on each mirror:

$$
\begin{aligned}
T & =\frac{2 P b}{c} \int\left[\tilde{u}_{0}(\vec{r})\right]^{2} r \cos \varphi r d r d \varphi \\
& =\frac{2 P b}{c} 2 \sum_{k=1} \alpha_{k} \int u_{0}(\vec{r}) u_{k}(\vec{r}) r \cos \varphi r d r d \varphi,
\end{aligned}
$$

where we have used Eq. (2.8). By inserting Eqs. (2.9) and (2.10), we obtain the following formulas for the tiltinduced torque to first order in $\alpha_{k}$ (first order in $\theta$ ):

$$
\begin{gathered}
T=\frac{2 \sqrt{2} P b,}{c} \times \sum_{k=1} \alpha_{k} I_{k}, \\
I_{k}=\int u_{0}(r) u_{k}(r) r^{2} d r .
\end{gathered}
$$

These formulas are valid for any FP cavity and, in particular, for FG, FM, CG, and CM cavities that interest us (of course, the modes $u_{k}(r)$ are different for different cavities).

In the sections below, we calculate the values for the coupling constants $\alpha_{k}$ and the overlap integrals $I_{k}$ for our four types of cavities. Our analysis for conventional spherical mirrors (FG and CG; Sec. III) is entirely analytical, whereas for any generic mirror shape, and $\mathrm{MH}$ mirrors, in particular, (FM and CM; Sec. IV), numerical treatment is required. We will test our numerical methods by applying them to FG and CG cavities and comparing with the analytical results.

\section{GAUSSIAN-BEAM (FG AND CG) CAVITIES}

We consider a cavity with identical spherical mirrors. We are interested in a symmetric tilt of the two mirrors by a small angle $\theta$ as shown on Fig. 1(b). In this case, the axis of the new mode $\tilde{u}_{0}(\vec{r})$ is displaced by a small distance $\delta x_{\text {sym }}$, but is still parallel to the old axis. The field distribution on each mirror will be unchanged, but shifted by $\delta x_{\text {sym }}$.

Spherical cavities have been studied thoroughly (see e.g [15]); their fundamental modes are the well-known GaussLaguerre modes (called in this paper FG and CG modes).
We will use these modes derive analytical formulas for $\alpha_{k}$ and $I_{k}$. The main axisymmetric and dipolar modes $\left[u_{0}(r)\right.$ and $\left.u_{1}(r)\right]$ are given by (see e.g. [16]):

$$
\begin{aligned}
u_{0}^{G}(r) & =\frac{\sqrt{2}}{r_{0}} e^{-r^{2} / 2 r_{0}^{2},} \\
u_{1}^{G}(r) & =\frac{\sqrt{2} r}{r_{0}^{2}} e^{-r^{2} / 2 r_{0}^{2}}, \\
r_{0} & =\frac{1}{\left(1-g^{2}\right)^{1 / 4}} .
\end{aligned}
$$

Here $r$ is the dimensionless radial coordinate (measured in units of $b$ ), $r_{0}$ is the dimensionless radius of the beam at the mirrors' surface (also in units of $b$ ), $g=1-L / R$ is the so-called $g$-parameter of the cavity, $L$ is the distance between the mirrors, and $R$ is the mirrors' radius of curvature [Fig. 1(a)]. (The intensity on the mirror is proportional to $e^{-r^{2} / r_{0}^{2}}$.)

For spherical mirrors the displacement of the optic axis $\delta x_{\text {sym }}$ is [see Fig. 1(b)]:

$$
\delta x_{\mathrm{sym}} \simeq \frac{R \theta}{b}=\frac{L \theta}{b(1-g)} .
$$

Next, we write down the main mode $\tilde{u}_{0}^{G}$ of the FP resonator with tilted mirrors and expand it to first order in $\delta x_{\text {sym }}$ :

$$
\begin{gathered}
\tilde{u}_{0}^{G}(\vec{r})=\frac{e^{-r_{\delta x}^{2} / 2 r_{0}^{2}}}{\sqrt{\pi}}, \\
r_{\delta x}^{2}=\left(r \cos \varphi-\delta x_{\mathrm{sym}}\right)^{2}+r^{2} \sin ^{2} \varphi \\
\tilde{u}_{0}^{G(\vec{r})}=u_{0}^{G(\vec{r})\left(1+\frac{r \delta x_{\mathrm{sym}} \cos \varphi}{r_{0}^{2}}\right)} \\
=u_{0}^{G(\vec{r})}+u_{0}^{\frac{\delta x_{\mathrm{sym}} \cos \varphi}{\sqrt{2 \pi} r_{0}} u_{1}^{G}(r)}+\underbrace{\frac{\delta x_{\mathrm{sym}}}{\sqrt{2} r_{0}}}_{\alpha_{1}^{G}} \underbrace{u_{1}^{G}(r) \cos \varphi}_{u_{1}^{G}(\vec{r}) .}
\end{gathered}
$$

As we can see, the only nonzero coupling constant is $\alpha_{1}^{G}$

$$
\alpha_{1}^{G}=\frac{L \theta(1+g)^{1 / 4}}{\sqrt{2} b(1-g)^{3 / 4}} .
$$

From Eqs. (2.14), (3.1), (3.2), and (3.3), we can easily calculate the only overlap integral we need for Gaussian beams:

$$
I_{1}^{G}=\int_{0}^{\infty} u_{0}^{G}(r) u_{1}^{G}(r) r^{2} d r=r_{0}=\frac{1}{\left(1-g^{2}\right)^{1 / 4}} .
$$

Substituting into Eq. (2.13) along with Eq. (3.8) we derive a 
final expression for the torque:

$$
T^{G}=\frac{2 P L}{c} \frac{\theta}{(1-g)} .
$$

This result, derived by a modal analysis, is in complete agreement with the result of the Sidles-Sigg geometrical analysis in its long-cavity limit (Section 5 of [9]). In their notation, the torque for the unstable configuration is

$$
T^{G}=-k_{-} \theta=\frac{2 P L}{c} \frac{\theta}{(1-g)},
$$

where $-k_{-}$is the negative eigenvalue of a torsional stiffness matrix (Eq. (23) of Section 5 in [9]). (Note that negative eigenvalues in the Sidles-Sigg analysis are associated with unstable configurations - the subject of interest in this paper.) Our perturbation method gives the exact result (to first order in $\theta$ ) for spherical mirrors, because the only contribution to the torque is from the lowest dipolar mode $u_{1}$. This is a property only for spherical mirrors and their Gaussian beams. As we will see in the following sections, for any generic mirror shapes, we have to calculate the contribution from all higher dipolar modes.

\section{MESA-BEAM (FM AND CM) CAVITIES: ANALYTICAL FORMULAS}

\section{A. Perfectly positioned mirrors [Fig. 1(a)]}

For any cavity with axisymmetric mirrors, and, in particular, MH mirrors, the main axisymmetric mode $u_{0}(\vec{r})$ and all dipolar modes $u_{k}(\vec{r})$ satisfy the integral eigenequations

$$
\begin{aligned}
& \int G\left(\vec{r}_{1}, \vec{r}_{2}\right) u_{0}\left(\vec{r}_{2}\right) d^{2} \vec{r}_{2}=\lambda_{0} u_{0}\left(\vec{r}_{1}\right), \\
& \int G\left(\vec{r}_{1}, \vec{r}_{2}\right) u_{k}\left(\vec{r}_{1}\right) d^{2} \vec{r}_{1}=\lambda_{k} u_{k}\left(\vec{r}_{2}\right),
\end{aligned}
$$

where $G, u_{0}, u_{k}, \vec{r}_{1}, \vec{r}_{2}$ are all dimensionless and the eigenvalue of the $k$ th dipolar mode $u_{k}$ is $\lambda_{k}$.

In the paraxial approximation, the kernel of the operator $G$ is the following (up to a trivial factor of $e^{i k L}$ due to phase accumulation along the arm length $L$, which we omit, thereby fixing a common overall phase factor in all the $\lambda_{k}$ ):

$$
\begin{aligned}
G\left(\vec{r}_{1}, \vec{r}_{2}\right) & =\frac{-i}{2 \pi} \exp \left[i\left(\frac{\left(\vec{r}_{1}-\vec{r}_{2}\right)^{2}}{2}-h_{1}\left(\vec{r}_{1}\right)-h_{2}\left(\vec{r}_{2}\right)\right)\right], \\
h_{1,2}(\vec{r}) & =k H_{1,2}(\vec{r}), \quad k=\frac{2 \pi}{\lambda} .
\end{aligned}
$$

Here $H_{1}\left(\vec{r}_{1}\right)$ and $H_{2}\left(\vec{r}_{2}\right)$ are the physical deviations of the mirrors' surfaces from a plane surface, which we assume to be the same, $H_{1}\left(\vec{r}_{1}\right)=H_{2}\left(\vec{r}_{2}\right)$ (identical mirrors).

\section{B. Symmetrically tilted mirrors [Fig. 1(b)]}

The tilt is equivalent to small deviations of each mirror's position from the unperturbed one:

$$
\begin{aligned}
& \delta h_{1}=k b r_{1} \cos \varphi_{1} \theta \quad \text { (left mirror) } \\
& \delta h_{2}=k b r_{2} \cos \varphi_{2} \theta \quad \text { (right mirror). }
\end{aligned}
$$

These tilts induce a coupling of all the dipolar modes $u_{1}, u_{2}, \ldots$ into the cavity's fundamental mode $\tilde{u}_{0}$, as shown in Eq. (2.8), though (as our numerical work will show) the coupling for the first dipolar mode is far greater than the others $\alpha_{1} I_{1} \gg \alpha_{k} I_{k}$ for $k=2,3, \ldots$

For simplicity, we will show the analysis only for the first dipolar mode $u_{1}$ ( $u_{01}$ in the conventional notation). The generalization for the higher dipolar modes is trivially obtained by replacing the subscript 1 by the desired dipolar mode's subscript $k$

The eigenvalue of the fundamental mode of the perturbed cavity $\tilde{\lambda}_{0}$ will slightly differ from $\lambda_{0}: \tilde{\lambda}_{0}=\lambda_{0}+$ $\Delta$. Thus, we have the following integral eigenequation for $\tilde{u}_{0}(\vec{r})$

$$
\begin{aligned}
\left(\lambda_{0}+\Delta\right)\left[u_{0}\left(\vec{r}_{1}\right)+\alpha_{1} u_{1}\left(\vec{r}_{1}\right)\right]= & \int G\left(\vec{r}_{1}, \vec{r}_{2}\right)\left[1-i \delta h_{1}\left(\vec{r}_{1}\right)\right. \\
& \left.-i \delta h_{2}\left(\vec{r}_{2}\right)\right]\left[u_{0}\left(\vec{r}_{2}\right)\right. \\
& \left.+\alpha_{1} u_{1}\left(\vec{r}_{2}\right)\right] d^{2} \vec{r}_{2} .
\end{aligned}
$$

This equation can be simplified by use of the eigenequation of the original unperturbed system (4.1):

$$
\begin{aligned}
& \Delta u_{0}\left(\vec{r}_{1}\right)+\left(\lambda_{0}+\Delta-\lambda_{1}\right) \alpha_{1} u_{1}\left(\vec{r}_{1}\right) \\
& =-i \int G\left(\vec{r}_{1}, \vec{r}_{2}\right)\left[\delta h_{1}\left(\vec{r}_{1}\right)+\delta h_{2}\left(\vec{r}_{2}\right)\right] u_{0}\left(\vec{r}_{2}\right) d^{2} \vec{r}_{2}
\end{aligned}
$$

Here, we have dropped a term proportional to $\delta h \alpha_{1} u_{1}$ in the integrand, since it is of second order in $\theta$.

Multiplying Eq. (4.6) by $u_{0}\left(\vec{r}_{1}\right)$ and integrating over $d^{2} \vec{r}_{1}$, one can find that, to first order in $\theta$, the correction $\Delta$ to the eigenvalue $\lambda_{0}$ is zero. Therefore, the correction of the eigenvalue has second order of smallness, so below we set $\Delta=0$.

Multiplying Eq. (4.6) by $u_{1}\left(\vec{r}_{1}\right)$ and integrating over $d^{2} \vec{r}_{1}$, one can find $\alpha_{1}$ :

$$
\left(\lambda_{0}-\lambda_{1}\right) \alpha_{1}=-i\left(\lambda_{0}+\lambda_{1}\right) \int u_{0}\left(\vec{r}_{1}\right) u_{1}\left(\vec{r}_{1}\right) \delta h_{1}\left(\vec{r}_{1}\right) d^{2} \vec{r}_{1},
$$

so

$$
\begin{aligned}
\alpha_{1} & =-\frac{i k b \theta\left(\lambda_{0}+\lambda_{1}\right)}{\sqrt{2}\left(\lambda_{0}-\lambda_{1}\right)} \underbrace{\int_{0}^{\infty} u_{0}(r) u_{1}(r) r^{2} d r}_{I_{1}} \\
& =-\frac{i L I_{1} \theta\left(\lambda_{0}+\lambda_{1}\right)}{\sqrt{2} b\left(\lambda_{0}-\lambda_{1}\right)} .
\end{aligned}
$$


Similarly for the higher dipolar modes

$$
\alpha_{k}=-\frac{i L I_{k} \theta\left(\lambda_{0}+\lambda_{k}\right)}{\sqrt{2} b\left(\lambda_{0}-\lambda_{k}\right)} .
$$

In order to calculate the numerical value of $\alpha_{k}$, we must solve the eigenequations (4.1) and (4.2) numerically for the eigenvalues $\lambda_{0}, \lambda_{k}$ and the corresponding eigenfunctions $u_{0}(r), u_{k}(r)$ (see Appendix for details). The value of the integral $I_{k}$ can be calculated numerically from Eq. (2.14).

Note that the formulas in this section are valid for any resonators with symmetric mirrors $H_{1}\left(r_{1}\right)=H_{2}\left(r_{2}\right)$ and very low diffraction losses, not just for mesa-beam resonators.

The coupling constant $\alpha_{k}$ is a measure of how much power is leaking out from the main resonant mode $u_{0}$ into a higher mode $u_{k}$. The torque exerted on the mirror is proportional to $\alpha_{k}$ [Eq. (2.13)] which depends on the relative location of the eigenvalues of the modes [Eq. (4.8)].

The best scenario would be if the cavity is designed so that $\lambda_{0}=-\lambda_{k}$ for some dominant mode $u_{k}$ so the contribution of this mode to the tilt instability is reduced to second order in the tilt angle $\theta$. However, there will still be a first-order contribution from the other modes that do not satisfy this property. As we shall see in Section VII, (luckily) the first dipolar mode of one of the configurations studied (nearly concentric Mexican-hat mirrors) has this property $\lambda_{0}=-\lambda_{k}$ almost satisfied and therefore this configuration is more stable compared than the others.

\section{NUMERICAL SOLUTIONS OF EIGENEQUATIONS}

We have solved the eigenEqs. (4.1) and (4.2) numerically using the scheme described in Appendix, for our four cavity configurations: FG, CG, FM, and CM. Recall that our nearly flat and nearly concentric cavities were chosen such that the intensity $u_{0}(r)^{2}$ and therefore $u_{0}(r)$ at the mirrors' surfaces are identical (FG and CG are the same and FM and CM are the same). We have found numerically for FM and CM (mesa beams) and for FG and CG (Gaussian beams, Sec. III) that $u_{k}(r)$ is also the same for the nearly concentric and nearly flat cases. The eigenfunctions $u_{0}$ and $u_{1}$ are shown in Fig. 2. The eigenvalues, by contrast, are different for nearly flat and nearly concentric cavities, so we have four sets of eigenvalues (FG, CG, FM, $\mathrm{CM}$ ), depicted in Fig. 3.

In our numerical solutions to the eigenequations (4.1) and (4.2), one of us (PS) found an interesting duality relation between nearly flat and nearly concentric configurations. This duality relation is satisfied for any generic mirror shape that satisfies the paraxial approximation. To within numerical error of less than 0.05 per cent, we found that a nearly concentric cavity, which has the same intensity profile as a nearly flat configuration, also has the same



FIG. 3. Eigenvalue spectrum in the complex plane. Note that all eigenvalues satisfy the duality relation, Eq. (5.2) $(n=0, m=$ 0 for $\lambda_{0}$, and $n=0, m=1$ for $\lambda_{1}$ ); see also [13].

mirror-shape correction as the nearly flat cavity, but with opposite sign ${ }^{2}$ :

$$
\delta h^{C}(r)=-\delta h^{F}(r) .
$$

Here $\delta h^{C}(r)$ is the deviation from concentric spherical shape, and $\delta h^{F}(r)$ is the deviation from flat shape. We also found, numerically, a unique mapping between the eigenvalues of these dual configurations:

$$
\lambda_{n m}^{C}=(-1)^{m+1}\left(\lambda_{n m}^{F}\right)^{*},
$$

for any pair of integers $n, m=0,1,2, \ldots$ In addition, all higher modes have the same intensity profiles at the mirrors' surfaces as their counterparts

$$
\left|u_{n m}^{C}\right|^{2}=\left|u_{n m}^{F}\right|^{2}
$$

for any integer $n, m=0,1, \ldots$.

Remarkably, our numerical calculations showed that these relations hold not just for mesa-beam cavities, but for all stable cavities that we explored (all mirror shapes $\delta h^{C, F}$, including cavities in which the deviations $\delta h^{C, F}$ from concentric spherical and flat shapes are large-as long as the paraxial approximation is valid).

This has led us to conjecture a duality relation between symmetric cavities with axisymmetric mirrors: for any two such cavities, $A$ and $B$, with

\footnotetext{
${ }^{2} \mathrm{M}$. Bondarescu [12] was the first to discover this fact numerically, though for a specific set of light beams: his "hyperboloidal beams".
} 


$$
h^{A}(r)+h^{B}(r)=\frac{r^{2}}{L}
$$

there exists a one-to-one correspondence between their eigenstates: they all have the same intensity profiles at the mirrors, while

$$
\lambda_{n m}^{A}=(-1)^{m+1}\left(\lambda_{n m}^{B}\right)^{*} .
$$

Chen and Savov, and independently Agresti and d'Ambrosio [13] have verified and generalized this conjecture analytically.

\section{STRENGTH OF THE TILT INSTABILITY FOR FG, CG, FM, AND CM CAVITIES}

We now have all the tools we need to compute the tiltinduced torque $T$ on the cavity's mirrors, for FG, CG, FM, and CM configurations. We shall evaluate $T$ for two sets of cavity parameters: the fiducial parameters used by d'Ambrosio et al. [1-3] and the advanced-LIGO baseline parameters (Table 1 in [11]).

The set of parameters for the fiducial cavity (see Sec. IVA(2) of [1] and Sec. IIIA of [2]) is:

$L=4 \mathrm{~km}$ - the length of the cavity.

$\lambda=1064 \mathrm{~nm}$ - the wavelength of the laser beam.

$k=2 \pi / \lambda$-the wave number associated with $\lambda$.

$b=\sqrt{L \lambda / 2 \pi}=2.603 \mathrm{~cm}$ - the natural diffraction length scale (Fresnel length).

$r_{\max }=16 \mathrm{~cm}$ - the radius of the mirrors' coated surfaces.

$g^{\mathrm{FG}}=0.952$ - the $g$-factor for the fiducial FG resonator (corresponding mirror radius of curvature $R=$ $83.33 \mathrm{~km})$.

$g^{\mathrm{CG}}=-0.952$ - the $g$-factor for the fiducial CG resonator (corresponding mirror radius of curvature $R=$ $2.05 \mathrm{~km})$.

$r_{0}=b /\left(1-g^{2}\right)^{1 / 4}=4.7 \mathrm{~cm}$ - the radius of the $\mathrm{FG}$ and $\mathrm{CG}$ beams at the mirrors.

$D=4 b=10.4 \mathrm{~cm}$ - the radius parameter of the FM and CM beams at the mirrors (see Sec. IIA and Sec. IVA(2) of [1]).

The above beam radii were chosen so as to make the diffraction losses be about $20 \mathrm{ppm}$ (ppm). More specifically, they are $23 \mathrm{ppm}$ for the FG and CG beams and 19 ppm for the FM and CM beams. ${ }^{3}$

From Eqs. (2.13), (3.8), and (3.9) we can calculate the integral $I_{1}^{G}$, the coupling constant $\alpha_{1}^{G}$, and the torque $T^{G}$ for the FG and CG cavities. Our results are shown in the second and third column of Table I.

We have already established an agreement between our analytically derived results using the modal analysis described in Sec. III and the Sidles-Sigg results derived from geometric considerations [9]. We can also test the numeri-

\footnotetext{
${ }^{3}$ We have deduced these diffraction losses from our numerical solutions of the cavity's eigenequation.
}

TABLE I. Comparison Between Analytical and Numerical Results for FG and CG Cavities; $\alpha_{1}$ is measured in units of $\left(\theta / 10^{-8}\right)$ and $T$ is in units of $(P b / c)\left(\theta / 10^{-8}\right)$.

\begin{tabular}{lllll}
\hline \hline & \multicolumn{2}{c}{ Analytical } & \multicolumn{2}{c}{ Numerical } \\
& \multicolumn{1}{c}{ FG } & \multicolumn{1}{c}{ CG } & \multicolumn{1}{c}{ FG } & \multicolumn{1}{c}{ CG } \\
\hline$I_{1}$ & 1.8075 & 1.8075 & 1.8073 & 1.8073 \\
$\alpha_{1}$ & 0.012526 & 0.00030802 & 0.012525 & 0.00030799 \\
$T$ & 0.064038 & 0.0015747 & 0.064023 & 0.0015743 \\
\hline \hline
\end{tabular}

cal first-order perturbation methods that we developed for arbitrary mirror shapes by applying them to our FG and CG cavities. By substituting our numerical results for $u_{0}^{\mathrm{FG}}=$ $u_{0}^{\mathrm{CG}}, u_{1}^{\mathrm{FG}}=u_{1}^{\mathrm{CG}}, \lambda_{0}^{\mathrm{FG}}, \lambda_{0}^{\mathrm{CG}}, \lambda_{1}^{\mathrm{FG}}$, and $\lambda_{1}^{\mathrm{CG}}$ into Eqs. (2.13), (3.9), and (4.7), we calculate the results shown in the last two columns of Table I. These numerical results all agree with our analytical results to within 0.05 per cent, thus validating our numerical methods.

As was found by Sidles and Sigg, the CG configuration is significantly less unstable than its nearly flat counterpart FG. The analytical analysis (first two columns in Table I predicts

$$
\frac{T^{\mathrm{FG}}}{T^{\mathrm{CG}}}=\frac{1+g^{\mathrm{FG}}}{1-g^{\mathrm{FG}}}=\frac{R^{\mathrm{FG}}}{R^{\mathrm{CG}}}=40.667,
$$

which is in agreement with the numerical result 40.667 (last two columns).

From the modal analysis applied to FG and CG cavities [Eqs. (2.13) and (4.7)], we deduce that, aside from factors that are the same for FG and CG,

$$
T^{G} \propto i \frac{\lambda_{0}^{G}+\lambda_{1}^{G}}{\lambda_{0}^{G}-\lambda_{1}^{G}}=\operatorname{cotan}\left(\frac{\phi_{01}^{G}}{2}\right) .
$$

Here, the equality holds because $|\lambda|=1$ for all modes (negligible diffraction losses) and $\phi_{01}^{G}$ is the phase separation between $\lambda_{0}^{G}$ and $\lambda_{1}^{G}$, i.e. the argument of $\lambda_{0}^{G} / \lambda_{1}^{G}$ (in Fig. 3 we show $\phi_{01}^{\mathrm{FM}}$ for the FM cavity). Thus, Eq. (6.2) is governed by the phase separation of the eigenvalues $\lambda_{0}^{G}$ and $\lambda_{1}^{G}$. As Fig. 3 shows the two eigenvalues for the FG configuration are very close to each other so $\operatorname{cotan}\left(\phi_{01}^{\mathrm{FG}} / 2\right) \gg 1$, whereas the phase separation of the eigenvalues for the CG configuration is close to $\pi$ so $\operatorname{cotan}\left(\phi_{01}^{\mathrm{CG}} / 2\right) \ll 1$. This explains why $T^{\mathrm{FG}} \gg T^{\mathrm{CG}}$.

Similarly to the above Gaussian analysis, we use our numerical results to compute the torques $T^{\mathrm{FM}}$ and $T^{\mathrm{CM}}$ for FM and CM cavities, respectively. In this case, we must include the contributions from higher order dipolar modes $\left(u_{1}, u_{2}\right.$, and $\left.u_{3}\right)$. From Eqs. (2.13), (2.14), and (4.8), we have calculated the integrals $I_{k}^{\mathrm{FM}}, I_{k}^{\mathrm{CM}}$, the coupling constants $\alpha_{k}^{\mathrm{FM}}, \alpha_{k}^{\mathrm{CM}}$, and the torques $T^{\mathrm{FM}}, T^{\mathrm{CM}}$ for the FM and $\mathrm{CM}$ cavities. Our results are shown in Table II. Note that the dominant contribution to the torque comes from the first dipolar mode, $k=1$; the higher modes give contributions of only a few per cent, at most. 
TABLE II. Numerical Results for FM and CM cavities; $\alpha_{k}$ is measured in units of $\left(\theta / 10^{-8}\right)$ and $T_{k}$ is in units of $(\mathrm{Pb} / \mathrm{c}) \times$ $\left(\theta / 10^{-8}\right)$.

\begin{tabular}{lcrcccc}
\hline \hline$k$ & $I_{k}^{\mathrm{FM}}$ & \multicolumn{1}{c}{$\alpha_{k}^{\mathrm{FM}}$} & $T_{k}^{\mathrm{FM}}$ & $I_{k}^{\mathrm{CM}}$ & \multicolumn{1}{c}{$\alpha_{k}^{\mathrm{CM}}$} & \multicolumn{1}{c}{$T_{k}^{\mathrm{CM}}$} \\
\hline 1 & 2.6464 & 0.04525 & 0.33867 & 2.6464 & 0.00018 & 0.00137 \\
2 & 0.1136 & 0.00009 & 0.00003 & 0.1136 & 0.00016 & 0.00005 \\
3 & -0.015 & -0.00000 & 0.00000 & -0.015 & -0.0002 & 0.00001 \\
Total & & & 0.33870 & & & 0.00143 \\
\hline \hline
\end{tabular}

For mesa-beam resonators, as in the case of Gaussianbeam resonators, the nearly flat configuration (FM) is far more unstable than its nearly concentric counterpart (CM)

$$
\frac{T^{\mathrm{FM}}}{T^{\mathrm{CM}}}=237 .
$$

In this case, the difference is even bigger than in the Gaussian case since the eigenvalues for the FM configuration are closer to each other on the unit circle (Fig. 3) than for the FG configuration and the phase separation of the eigenvalues for the CM configuration is even closer to $\pi$ than the phase separation of the eigenvalues for the $\mathrm{CG}$ configuration (Fig. 3).

In Table III, we compare all four configurations FG, CG, FM, and CM, normalized by $T^{\mathrm{CG}}$. For nearly flat resonators, going from a Gaussian-beam to a mesa-beam configuration increases the strength of the instability by about a factor 5. There are two effects contributing to this increase as we can see from the following relation (in which we focus on the dominant, $k=1$ contribution):

$$
\begin{aligned}
\frac{T^{M}}{T^{G}} & =\frac{\alpha_{1}^{M} I^{M}}{\alpha_{1}^{M} I^{M}}=\left(\frac{\lambda_{0}^{M}+\lambda_{1}^{M}}{\lambda_{0}^{G}+\lambda_{1}^{G}}\right)\left(\frac{\lambda_{0}^{G}-\lambda_{1}^{G}}{\lambda_{0}^{M}-\lambda_{1}^{M}}\right)\left(\frac{I^{M}}{I^{G}}\right)^{2} \\
& =\frac{\operatorname{cotan}\left(\phi_{01}^{M} / 2\right)}{\operatorname{cotan}\left(\phi_{01}^{G} / 2\right)}\left(\frac{I^{M}}{I^{G}}\right)^{2} .
\end{aligned}
$$

In the case of the nearly flat configurations both phase differences are small and since $\phi_{01}^{\mathrm{FM}}<\phi_{01}^{\mathrm{FG}}$ (see Fig. 3),

$$
\frac{\operatorname{cotan}\left(\phi_{01}^{\mathrm{FM}} / 2\right)}{\operatorname{cotan}\left(\phi_{01}^{\mathrm{FG}} / 2\right)}>1 \text {. }
$$

This effect is amplified by the second ratio because of the higher overlap between the two eigenstates in the case of mesa beams than for Gaussian beams. This is manifested in the higher value of $I^{\mathrm{FM}}=2.65$ compared to $I^{\mathrm{FG}}=1.87$

TABLE III. Comparison between different configurations of a fiducial optical cavity. The torques due to light pressure (when tilt angle $\theta$ and circulating power $P$ are the same) are normalized such that $T^{\mathrm{CG}}=1$.

\begin{tabular}{lcc}
\hline \hline & Nearly Flat Cavity & Nearly Concentric Cavity \\
\hline$G$-Beam & $T^{\mathrm{FG}}=40.7$ & $T^{\mathrm{CG}}=1.0$ \\
$M$-Beam & $T^{\mathrm{FM}}=215$ & $T^{\mathrm{CM}}=0.91$ \\
\hline \hline
\end{tabular}

(compare the overlaps between each pair of modes in Fig. 2(a) and 2(b)].

For nearly concentric resonators, going from Gaussianbeam resonators to mesa-beam resonators weakens the net instability: $T^{\mathrm{CM}} / T^{\mathrm{CG}}=0.91$. In this case, the difference in the overlaps of the eigenstates is unchanged, but the phase differences are close to, but less than $\pi$. Since $\phi_{01}^{\mathrm{CM}}>\phi_{01}^{\mathrm{CG}}$ (again look at the separation of each set of eigenvalues on the unit circle for the CG and CM configurations in Fig. 3),

$$
\frac{\operatorname{cotan}\left(\phi_{01}^{\mathrm{CM}} / 2\right)}{\operatorname{cotan}\left(\phi_{01}^{\mathrm{CG}} / 2\right)}<1
$$

The two effects counteract each other and for this choice of parameters the net result is in favor of the CM-Beam resonator. The comparison between the torques for nearly flat and nearly concentric cavities is straightforward using Eq. (6.4) and the duality relation (see Eq. (5.2) and Ref. [13]).

In our formulation of the perturbation theory, we account for effects scaled to first order in the tilt angle $\theta$. We assume small mode mixing $\alpha_{k} \ll 1$ in order for the perturbation method to work. From our numerical results (Table II), we see that $\alpha_{k} \ll 1$ requires the angular orientation of the cavity mirrors be controlled to $\theta<10^{-8}$.

The contributions $T_{k}$ of the higher order dipolar modes $k=2,3, \ldots$ to the torque can be understood by studying the analog of Eq. (6.4). From the relative locations of the eigenvalues along the unit circle and the overlapping of the eigenmodes, it is easy to show that $T_{k}$ 's are monotonically decreasing, $T_{1}>T_{2}>T_{3} \ldots$ Thus, we accept the contribution from the highest dipolar mode $u_{3}$ in our calculation, including the numerical error, as the maximum error of the method due to neglecting the higher order dipolar modes. In this way, we conclude that the error in our total torque in the case of the CM cavity is less than 1 per cent. In the case of the FM cavity the error of the method is practically of order of the numerical error, so it is less than 0.1 per cent.

For another comparison, we perform the same calculations for the baseline design of advanced LIGO (Table 1 in [11]). The baseline parameters were chosen such that the beam radius at the mirrors ${ }^{5}$ in the case of spherical mirrors is $4.24 \mathrm{~cm}$, corresponding to diffraction losses of $10 \mathrm{ppm}$. The MH-mirror configurations are designed to have about the same diffraction losses. The resulting baseline parameters are:

$r_{\text {max }}=(15.7-0.8) \mathrm{cm}=14.9 \mathrm{~cm}$ - the radius of the coated mirrors' surfaces.

$g^{\mathrm{FG}}=0.9265$ - the $g$-factor for FG resonator (corresponding mirror radius of curvature $R=54.44 \mathrm{~km}$ ).

\footnotetext{
${ }^{4}$ Currently, the control system of the initial LIGO interferometers operates with accuracy $\theta \simeq 10^{-7}$; an accuracy $\theta \simeq 10^{-8}$ is planned for advanced LIGO interferometers [17].

${ }^{5}$ Note that our definition for the beam radius at the mirrors differs from [11] by factor of $\sqrt{2}$.
} 
TABLE IV. Comparison between different configurations of a cavity with parameters of the current baseline design for advanced LIGO. The torques due to light pressure (when tilt angle $\theta$ and circulating power $P$ are the same) are normalized such that $T^{\mathrm{CG}}=1$.

\begin{tabular}{lcc}
\hline \hline & Nearly Flat Cavity & Nearly Concentric Cavity \\
\hline$G$-Beam & $T^{\mathrm{FG}}=26.2$ & $T^{\mathrm{CG}}=1.0$ \\
$M$-Beam & $T^{\mathrm{FM}}=96$ & $T^{\mathrm{CM}}=0.91$ \\
\hline \hline
\end{tabular}

$g^{\mathrm{CG}}=-0.9265$ - the $g$-factor for CG resonator (corresponding mirror radius of curvature $R=2.076 \mathrm{~km}$ ).

$r_{0}=b /\left(1-g^{2}\right)^{1 / 4}=4.24 \mathrm{~cm}$ - the radius of the Gaussian beam at the mirrors.

$D=3.3 b=8.58 \mathrm{~cm}$ - the radius parameter of the mesa beam at the mirrors.

Table IV contains the final results for these baseline parameters (including the sum of the contributions to the torques from the first three dipolar modes). Again, the least unstable configuration, and thus the easiest to control against tilt, is the nearly concentric mesa-beam (CM) resonator.

\section{CONCLUSIONS}

In this paper, we have investigated the problem of the tilt instability for several possible configurations of advanced LIGO. By using semianalytical and numerical techniques, we came to the conclusion that concentric Mexican-hat mirrors supporting mesa beams suffer the least instability and, therefore, pose the least problem to control the tilt instability. As Table IV shows, by switching from conventional Gaussian-beam cavities to concentric mesa-beam cavities, the instability to symmetric tilt will be reduced (dramatically compared to a flat Gaussian-beam cavity and moderately compared to a concentric Gaussian-beam cavity). Furthermore the sensitivity of the interferometer will improve significantly due to the reduced thermal noise (see e.g. Table I in [3] and also [4-6,18,19]).

We have also reported on a unique duality relation between the eigenspectra of optical cavities with mirror shapes corrected from plane-parallel and from concentric spherical surfaces. The one-to-one mapping of the eigenvalues and the eigenmodes can be a very powerful tool in solving other problems involving modal analysis of optical cavities. In a companion paper [13], we provide an analytical proof and generalization of this conjecture.

\section{ACKNOWLEDGMENTS}

We are very grateful to Kip Thorne who could be regarded as the "father" of this paper; he posed the problem; it was his idea to use CM beams to reduce the tilt instability; and he took part in very fruitful discussions and gave us useful advice. We thank Thorne and Yanbei Chen for helpful advice about the wording of this paper. For useful scientific discussions, we thank Thorne, Chen, Juri Agresti, Mihai Bondarescu, Erika d'Ambrosio, Poghos Kazarian, and Richard O'Shaughnessy. The research reported in this paper was supported in part by National Science Foundation grants No. PHY-0098715 and PHY- 0099568, by Russian Foundation for Fundamental Research grants No. 03-02-16975-a and by contracts No. 40.02.1.1.1.1137 and No. 40.700.12.0086 of the Industry and Science Ministry of Russia.

\section{APPENDIX: NUMERICAL SOLUTIONS OF CAVITY EIGENEQUATIONS}

In order to generate the set of basis solutions needed to construct perturbation theory for a cavity with arbitrary mirror shapes, we must numerically solve an integral eigenequation. We have done so using the following method, based on earlier work by O'Shaughnessy (Sec. VB of [3]).

Since the mirrors are axisymmetric $[h(\vec{r})=h(r)]$, we can decouple the angular and radial dependences in the eigenequations. In the numerical implementation of the eigensolver we used the following definition:

$$
u_{n m}(\vec{r})=u_{n m}(r) e^{-\mathrm{im} \varphi}, \quad m=0,1,2, \ldots
$$

Note that, for $m=0,1$, this definition of the fundamental radial mode $u_{0}(r)$ and the dipolar radial mode $u_{1}(r)$ differ from the definitions in Eqs. (2.9) and (2.10). However, after solving the eigenequations, all modes are renormalized by numerically computing the integrals in Eqs. (2.11) and (2.12), so at the end we have radial modes defined as in Eqs. (2.9) and (2.10). The resulting $u_{k}$ are the radial modes we need for computing $I_{k}$ in Eq. (2.14). By plugging Eq. (A1) into Eqs. (4.1) and (4.2) and integrating over the azimuthal angle, we can reduce the eigenproblem to a onedimensional integral equation

$$
\begin{aligned}
\lambda_{n m} u_{n m}\left(r_{1}\right) & =\int G_{m}\left(r_{1}, r_{2}\right) u_{n m}\left(r_{2}\right) r_{2} d r_{2}, \\
G_{m}\left(r_{1}, r_{2}\right) & =(-i)^{m+1} J_{m}\left(r_{1} r_{2}\right) \exp \left[i\left(\frac{\left(r_{1}^{2}+r_{2}^{2}\right)}{2}-2 h(r)\right)\right],
\end{aligned}
$$

where $J_{m}$ is the Bessel function of the first kind and order $m$.

We discretize space along the mirrors' radial direction in a uniform grid

$$
r_{j}=j r_{\max } /(N-1), \quad j=0,1, \ldots, N-1 .
$$

We define the matrix $G_{(m) i j}=G_{m}\left(x_{i}, x_{j}\right)$, the eigenvectors $u_{(n) j}=u_{n}\left(x_{j}\right)(m, n$ label the mode and $i, j$ are indices to access the matrices' and vectors' components), and we approximate the integration by a simple quadrature rule. Then the integral eigenproblem reduces to a matrix eigenvalue problem: 
$\lambda_{n m} \vec{u}_{n}=\hat{M}_{m} \vec{u}_{n} \quad$ with $\quad \hat{M}_{(m) i j}=\frac{r_{\max }^{2} j}{N-1} G_{(m) i j}$.
This equation can be solved for $\lambda_{n m}$ and $\vec{u}_{n}$ by any standard matrix eigensolution software package.
[1] E. d'Ambrosio, R. O'Shaughnessy, S. Strigin, K. Thorne, and S. Vyatchanin, gr-qc/0409075.

[2] E. D'Ambrosio, R. O'Shaughnessy, S. Strigin, K. Thorne, and S. Vyatchanin, LIGO Report No. LIGO-T030009-00 (unpublished); available at http://docuserv.ligo.caltech. edu/.

[3] R. O'Shaughnessy, S. Strigin, and S. Vyatchanin, gr-qc/ 0409050.

[4] J. Agresti (2004), http://www.ligo.caltech.edu/docs/T/ T040225-00.pdf.

[5] J. Agresi and R. DeSalvo (2005), http://www.ligo.caltech. edu/docs/G/G050041-00/.

[6] J. Agresti (unpublished).

[7] G. Lovelace (unpublished).

[8] D. Sigg, "Angular Instability in High Power FP Cavities" (2003), http://www.ligo.caltech.edu/docs/T/T030120-00. pdf.

[9] J. Sidles and D. Sigg, Phys. Lett. A 354, 167 (2006).

[10] V. Braginsky and A. Manukin, Measurement of Weak Forces in Physics Experiments (University of Chicago
Press, Chicago, 1977), pp. 25-39.

[11] D. Shoemaker, "Advanced LIGO: Context and Overview (Proposal to the NSF)" (2003), URL http://www.ligo. caltech.edu/docs/M/M030023-00/.

[12] M. Bondarescu and K. S. Thorne, Phys. Rev. D 74, 082003 (2006).

[13] J. Agresti, E. D’Ambrosio, Y. Chen, and P. Savov, gr-qc/ 0511062.

[14] S. Vyatchanin, "Estimate of Angular Instability for Mexican-Hat and Gaussian Modes of a Fabry-Perot Interferometer', (2003), http://www.ligo.caltech.edu/docs/ T/T030272-00.pdf.

[15] A. Siegman, An Introduction to Lasers and Masers (McGraw-Hill, New York, 1971), Ch. 8.

[16] A. Siegman, Lasers (Univ. Science Book, Mill Valley, CA, 1996), Ch. 19.

[17] Daniel Sigg (private communication).

[18] V. Braginsky, M. Gorodetsky, and S. Vyatchanin, Phys. Lett. A 264, 1 (1999).

[19] Y. T. Liu and K. Thorne, Phys. Rev. D 62, 122002 (2000). 